\title{
Rayleigh scattering in the transit spectrum of HD 189733b
}

\author{
A. Lecavelier des Etangs ${ }^{1,2}$, F. Pont ${ }^{3}$, A. Vidal-Madjar ${ }^{1,2}$, and D. Sing ${ }^{1,2}$ \\ 1 CNRS, UMR 7095, Institut d'Astrophysique de Paris, 98bis boulevard Arago, 75014 Paris, France \\ e-mail: lecaveli@iap.fr \\ 2 UPMC Univ. Paris 6, UMR 7095, Institut d'Astrophysique de Paris, 98bis boulevard Arago, 75014 Paris, France \\ 3 Physikalisches Institut, University of Bern, Sidlerstrasse 5, 3012 Bern, Switzerland
}

Received 11 January 2008 / Accepted 18 February 2008

\begin{abstract}
The transit spectrum of the exoplanet HD 189733b has recently been obtained between 0.55 and $1.05 \mu \mathrm{m}$. Here we present an analysis of this spectrum. We develop first-order equations to interpret absorption spectra. In the case of HD 189733b, we show that the observed slope of the absorption as a function of wavelength is characteristic of extinction proportional to the inverse of the fourth power of the wavelength $\left(\propto \lambda^{-4}\right)$. Assuming an extinction dominated by Rayleigh scattering, we derive an atmospheric temperature of $1340 \pm 150 \mathrm{~K}$. If molecular hydrogen is responsible for the Rayleigh scattering, the atmospheric pressure at the planetary characteristic radius of 0.1564 stellar radius must be $410 \pm 30$ mbar. However the preferred scenario is scattering by condensate particles. Using the Mie approximation, we find that the particles must have a low value for the imaginary part of the refraction index. We identify $\mathrm{MgSiO}_{3}$ as a possible abundant condensate whose particle size must be between $\sim 10^{-2}$ and $\sim 10^{-1} \mu \mathrm{m}$. For this condensate, assuming solar abundance, the pressure at 0.1564 stellar radius is found to be between a few microbars and few millibars, and the temperature is found to be in the range 1340-1540 K, and both depend on the particle size.
\end{abstract}

Key words. stars: planetary systems - scattering - techniques: spectroscopic - stars: individual: HD 189733b

\section{Introduction}

Transiting extrasolar planets, like HD 189733b offer unique opportunities to scrutinize their atmospheric content (e.g., Charbonneau et al. 2002; Vidal-Madjar et al. 2003). In particular, primary transits can reveal tenuous quantities of gas or dust by spectral absorption leading to variations in the apparent planet size as a function of the wavelength. HD $189733 \mathrm{~b}$ is presently the best target for such studies because of its nearby and bright host star, its large absorption depth in its transit light curve (Bouchy et al. 2005), and a short period (Hébrard \& Lecavelier des Etangs 2006).

Using transit observations with the ACS camera of the Hubble Space Telescope, the apparent radius of HD 189733b has been measured from 0.55 to 1.05 microns, thus providing the "transmission spectrum" of the atmosphere (Pont et al. 2008). In this wavelength range, strong features of abundant atomic species and molecules were predicted but not detected, leading Pont et al. (2008) to the conclusion that a haze of sub-micron particles is present in the upper atmosphere of the planet.

Here we use these measurements of the planet radius as a function of the wavelength from 550 to $1050 \mathrm{~nm}$. In Sect. 2 we describe how a transmission spectrum can be interpreted to derive basic quantities. We then estimate the temperature of HD 189733 b from fit to the ACS spectrum and show that the observed absorption can be caused by Rayleigh scattering (Sect. 3). Several species that are possibly responsible for the Rayleigh scattering are discussed, and the corresponding pressure at the absorption level are estimated in Sect. 4.

\section{Interpreting a transit spectrum}

\subsection{Planet radius as a function of atmospheric content}

To interpret transit spectra, one can use detailed atmosphere models that include temperature, pressure, and composition as a function of the altitude and numerically integrate the radiative transfer equations to obtain a theoretical spectrum to compare to the observations (e.g., Ehrenreich et al. 2006). Here we propose another approach by deriving first-order equations to obtain a better feeling for the basic quantities that can be obtained from these measurements.

Following Fortney's (2005) derivation of the path length, the optical depth, $\tau$, in a line of sight grazing the planetary limb at an altitude $z$ is given by $\tau(\lambda, z) \approx \sigma(\lambda) n(z) \sqrt{2 \pi R_{\text {planet }} H}$, where $R_{\text {planet }}$ is the assumed planet radius, $H$ the atmosphere scale height, and $n(z)=n_{(z=0)} \exp (-z / H)$ the volume density at the altitude $z$ of the main absorbent with a cross section $\sigma(\lambda)$. For a temperature $T, H$ is given by $H=k T / \mu g$, where $\mu$ is the mean mass of atmospheric particles taken to be 2.3 times the mass of the proton, and $g$ the gravity. Using planetary transit measurements, a fit to the light curve provides the ratio of the effective planetary radius as a function of wavelength to the stellar radius: $R_{\mathrm{p}}(\lambda) / R_{*}$. Here we define $\tau_{\text {eq }}$ by the optical depth at altitude $z_{\text {eq }}$ such that a sharp occulting disk of radius $R_{\text {planet }}+z_{\text {eq }}$ produces the same absorption depth as the planet with its translucent atmosphere; in other words, $\tau_{\mathrm{eq}}$ is defined by $R_{\mathrm{p}}(\lambda)=R_{\text {planet }}+z\left(\tau=\tau_{\text {eq }}\right)$. Using a model atmosphere, and numerically integrating over the whole translucent atmosphere, we can calculate the effective planet radius, and then obtain the corresponding optical thickness at the effective radius, $\tau_{\text {eq. }}$. For 


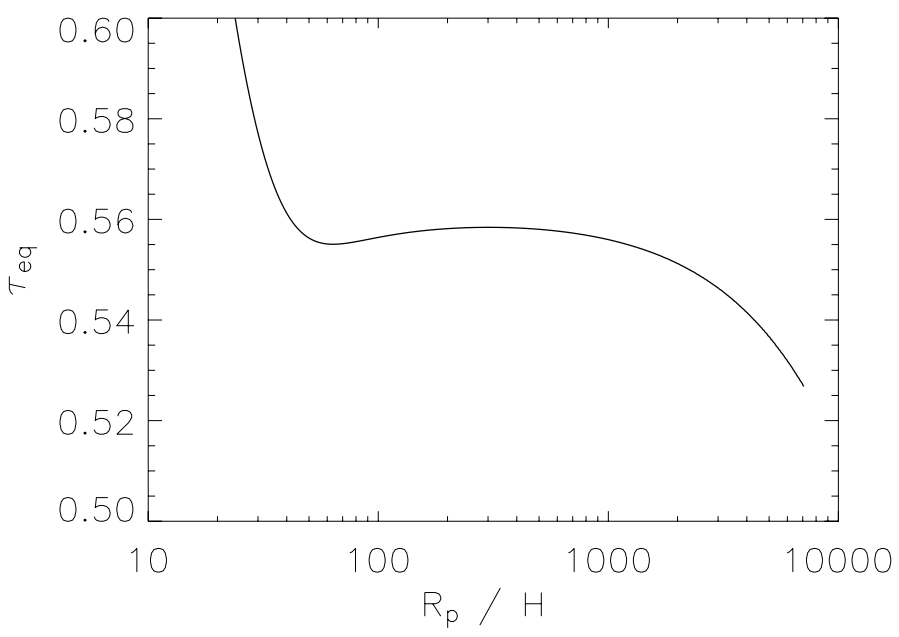

Fig. 1. Plot of $\tau_{\mathrm{eq}}$ as a function of the ratio of the planet radius to the atmosphere scale height.

various atmospheric scale heights, we calculate $\tau_{\text {eq }}$ by numerical integration. For a wide range of atmospheric scale height, provided that $R_{\mathrm{p}} / H$ is between $\sim 30$ and $\sim 3000$, the resulting $\tau_{\mathrm{eq}}$ is roughly constant at a value $\tau_{\text {eq }} \approx 0.56$ (Fig. 1 ). In the case of HD $189733 \mathrm{~b}, R_{\mathrm{p}} / H$ varies between 280 and 560 when the temperature varies from 1000 to $2000 \mathrm{~K}$; therefore, the approximation of a constant $\tau_{\text {eq }}$ at 0.56 fully applies. This demonstrates that, for a given atmospheric structure and composition, estimating the altitude at which $\tau=\tau_{\text {eq }}=0.56$ is all that is needed to calculate the effective radius of the planet at a given wavelength.

\subsection{Consequences}

For a given atmospheric structure and composition, the effective altitude of the atmosphere at a wavelength $\lambda$ is calculated by finding $z\left(\tau=\tau_{\text {eq }}, \lambda\right)$, which solves the equation $\tau(\lambda, z)=\tau_{\text {eq }}$. Using the quantities defined above, the effective altitude $z$ is given by

$z(\lambda)=H \ln \left(\xi_{\mathrm{abs}} P_{z=0} \sigma_{\mathrm{abs}}(\lambda) / \tau_{\mathrm{eq}} \times \sqrt{2 \pi R_{\mathrm{p}} / k T \mu g}\right)$,

where $\sigma_{\text {abs }}$ and $\xi_{\text {abs }}$ are the cross section and abundance of the dominant absorbing species.

If the variation of the cross section as a function of wavelength is known, the observation of the altitude as a function of wavelength allows the derivation of $H$ and, therefore, of the temperature $T$, given by:

$T=\frac{\mu g}{k}\left(\frac{\mathrm{d} \ln \sigma}{\mathrm{d} \lambda}\right)^{-1} \frac{\mathrm{d} z(\lambda)}{\mathrm{d} \lambda}$.

Using Eq. (1), the partial pressure of the main absorbent at the reference altitude is estimated by

$\xi_{\mathrm{abs}} P_{z=0}=\tau_{\mathrm{eq}} / \sigma_{\mathrm{abs}}\left(\lambda_{z=0}\right) \times \sqrt{k T \mu g / 2 \pi R_{\mathrm{p}}}$,

where $\lambda_{z=0}$ is the wavelength at which the effective planetary radius corresponds to (or is used to define) $z=0$. We note that there is a degeneracy between the abundance and the total pressure in the atmosphere. From the measurement of the effective radius using transit spectroscopy, one needs to assume an abundance of the absorbent to derive the pressure, or alternatively to assume a pressure to derive the abundance.

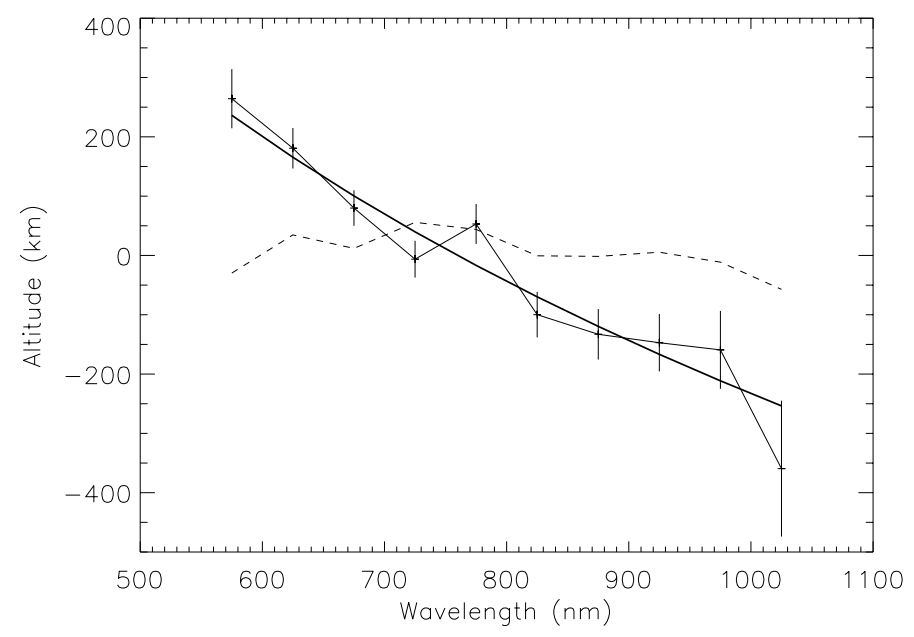

Fig. 2. Plot of the altitude in the atmosphere of HD 189733 b corresponding to the measured planetary radius as a function of wavelength. Here the zero altitude is defined for a planetary radius of 0.1564 times the stellar radius. The measured values with error bars are those of Pont et al. (2008). The fit to the data (thick line) is obtained assuming an extinction proportional to $\lambda^{-4}$ and for an atmospheric temperature of $1340 \mathrm{~K}$. Fits using $\mathrm{H}_{2}$ Rayleigh scattering or $\mathrm{MgSiO}_{3}$ grains with sizes between 0.01 and $0.1 \mu \mathrm{m}$ give the same plots. Larger grain diameters are not consistent with the observations (e.g., $0.4 \mu \mathrm{m}$, shown with dotted line).

In summary, the effective planetary radius is characteristic of the pressure and abundance, and the variation in this radius as a function of wavelength is characteristic of the temperature.

\section{The atmospheric temperature in HD 189733b}

In HD 189733b, the plot of altitude as a function of the wavelength shows an increase in absorption toward shorter wavelengths (Fig. 2). Using Eq. (2) and assuming a scaling law for the cross section in the form $\sigma=\sigma_{0}\left(\lambda / \lambda_{0}\right)^{\alpha}$, the slope of the planet radius as a function of the wavelength is given by $\mathrm{d} R_{\mathrm{p}} / \mathrm{d} \ln \lambda=\mathrm{d} z / \mathrm{d} \ln \lambda=\alpha H$. Therefore, we have

$\alpha T=\frac{\mu g}{k} \frac{\mathrm{d} R_{\mathrm{p}}}{\mathrm{d} \ln \lambda}$.

For HD 189733b, the slope is measured to be $472 \mathrm{~km} \pm 42$ (stat.) \pm 106 (syst.) from 600 to $1000 \mathrm{~nm}$ (Pont et al. 2008). This corresponds to $\mathrm{d} R_{\mathrm{p}} / \mathrm{d} \ln \lambda \approx-920 \mathrm{~km}$. We therefore obtain $\alpha T \approx-5840 \mathrm{~K}$. The temperature in HD $189733 \mathrm{~b}$ is determined to be in the range 900-1500 K (Deming et al. 2006; Knutson et al. 2007); therefore, $\alpha$ is found to be close to $\alpha \approx-4$, which is typical of Rayleigh scattering.

Assuming $\alpha=-4$ as expected for Rayleigh scattering and using Eq. (2), we derive a temperature

$T=1460 \pm 130$ (stat.) \pm 330 (syst.) K,

where (stat.) and (syst.) correspond to statistical and systematics error bars on $\mathrm{d} R_{\mathrm{p}}$, as quoted in Pont et al. (2008).

For condensates, the scale height $H_{\mathrm{c}}$ is often found to be significantly smaller than the gaseous scale height $\left(H_{\mathrm{c}} \sim H / 3\right)$ (Ackerman \& Marley 2001; Fortney 2005). If this were the case, because of the observed slope in the spectrum, the temperature (or $\alpha$ ) should be about three times higher than found above, which seems unlikely. We therefore conclude that, if produced by dust condensates, the observed transit spectrum of HD 189733 b shows that these condensates must be unusually 
well-mixed vertically with the atmospheric gas with a similar scale height.

\section{Rayleigh scattering}

\subsection{Molecular hydrogen}

One possible carrier of the Rayleigh scattering is the most abundant molecule, molecular hydrogen. The effective altitude at which the Rayleigh scattering of molecular hydrogen dominates only depends on the mean density. Therefore, measurements of the altitude in the regime where Rayleigh scattering by $\mathrm{H}_{2}$ dominates over other absorbents allows the determination of not only the temperature, but because the abundance of $\mathrm{H}_{2}$ is close to 1 $\left(\xi_{\mathrm{H}_{2}} \sim 1\right)$ also the total density and consequently the total pressure at the reference zero altitude.

Following Eq. (3), the pressure $P_{0}$ at the altitude corresponding to the radius at wavelength $\lambda_{0}$ is

$P_{0}=\tau_{\text {eq }} / \sigma_{0} \times \sqrt{k T \mu g / 2 \pi R_{\mathrm{p}}}$,

where $\sigma_{0}$ is the Rayleigh scattering cross section at $\lambda_{0}$. Using the refractive index of molecular hydrogen $(r-1)=1.32 \times 10^{-4}$, one derives $\sigma_{0}=2.52 \times 10^{-28} \mathrm{~cm}^{2}$ at $\lambda_{0}=750 \mathrm{~nm}$ (Bates 1984; Naus $\&$ Ubachs 2000). Using these numbers one finds $P_{0} \approx 400 \mathrm{mbar}$.

A fit with two free parameters (temperature and pressure) and assuming that Rayleigh scattering by molecular hydrogen dominates leads to a very good fit to the data published by Pont et al. (2008) with a $\chi^{2}$ equals 9.8 for 8 degrees of freedom. We thus obtain a temperature of $T=1340 \pm 150 \mathrm{~K}$ and a pressure at $z=0$ (defined by $R_{\mathrm{p}} / R_{*}=0.1564$ ) of $P=410 \pm 30$ mbar.

\subsection{Condensate}

Another possible carrier of the Rayleigh scattering is haze condensate typically smaller than the wavelength.

Assuming that the absorption measured between 550 and $1050 \mathrm{~nm}$ is due to particle condensate, we calculate the transmission spectrum using the Mie approximation. In transmission spectroscopy, given the small solid angle defined by the star seen from the planet, we can use the single scattering approximation. The glow produced by off-axis scattering is also negligible (Hubbard et al. 2001). In this configuration, the Mie extinction efficiency $\left(Q_{\text {ext }}\right)$ is used and calculated as the summation of the absorption efficiency $\left(Q_{\text {abs }}\right)$ and scattering efficiency $\left(Q_{\text {sca }}\right)$.

Rayleigh scattering in the form $\lambda^{-4}$ is obtained only for particle sizes $(a)$ much smaller than the wavelength $(2 \pi a \ll \lambda)$. In this last case, the absorption and scattering efficiencies are given by the approximation: $Q_{\mathrm{abs}}=(8 \pi a / \lambda) \mathfrak{J}\left(\left(\boldsymbol{n}^{2}-1\right) /\left(\boldsymbol{n}^{2}+2\right)\right)$, and $Q_{\text {sca }}=8 / 3(2 \pi a / \lambda)^{4} \mathfrak{R}\left(\left(\left(\boldsymbol{n}^{2}-1\right) /\left(\boldsymbol{n}^{2}+2\right)\right)^{2}\right)$, where $\boldsymbol{n}=n+\mathrm{i} k$ is the complex refraction index of the condensate.

We see from the two last equations that Rayleigh scattering dominates the transit spectrum only if $Q_{\text {abs }} \ll Q_{\text {sca }}$. Assuming that the imaginary part of the refraction index is much smaller than the real part, we find that this condition occurs for particle size, $a$, larger than a minimum size:

$a \gg a_{\min }=0.331 \sqrt[3]{\frac{n k}{\left(n^{2}-1\right)^{2}}} \lambda$.

For many plausible dust condensates (like $\mathrm{Mg}_{2} \mathrm{SiO}_{4}, \mathrm{MgFeSiO}_{4}$, $\mathrm{O}$-deficient silicates, etc.), the imaginary part of the refraction index is about $k \sim 0.1$, and the real part is in the range $n \sim 1.5-2.0$

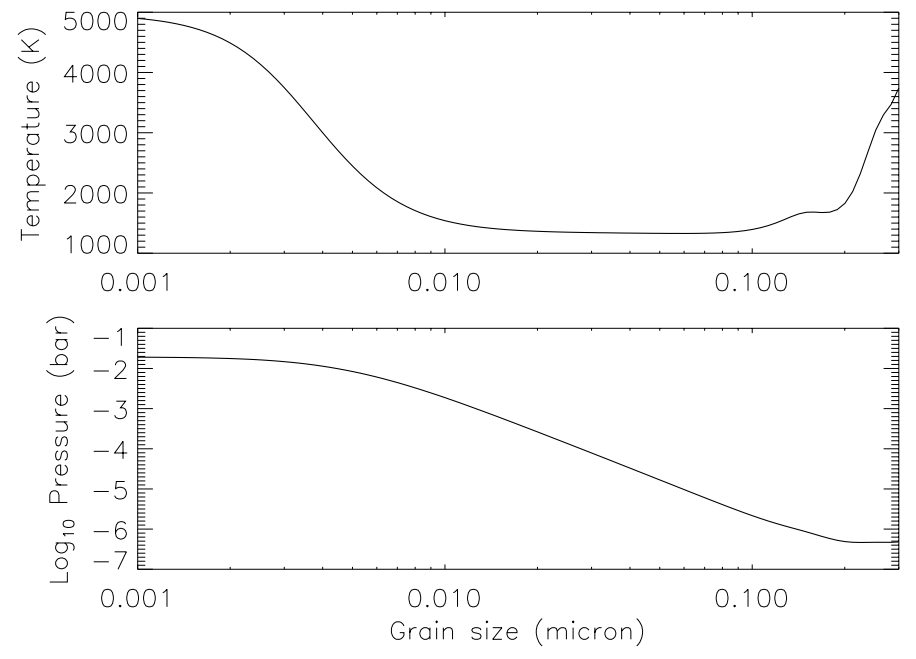

Fig. 3. Plot of the temperature and pressure at zero altitude as a function of the size of $\mathrm{MgSiO}_{3}$ grains needed to obtain a satisfactory fit to the data as in Fig. 2. Here we assume solar abundance for magnesium atoms to derive the grain abundance. For particles of $0.03 \mu \mathrm{m}$, temperature is found to be the same as with $\mathrm{H}_{2}$ Rayleigh scattering : $T=1340 \pm 150 \mathrm{~K}$. At 0.01 and $0.1 \mu \mathrm{m}$ temperature increases slightly to $T=1540 \pm 170 \mathrm{~K}$ and $T=1390 \pm 150 \mathrm{~K}$, respectively. For particles smaller than $\sim 0.01 \mu \mathrm{m}$ and larger than $\sim 0.1 \mu \mathrm{m}$, Rayleigh scattering does not dominate the extinction $(-\alpha \ll 4)$, and the temperature must be higher to fit the slope of the measured radius as a function of wavelength. At $0.3 \mu$ the fit is bad with $\chi^{2}=22.3$ for 8 degrees of freedom; $\chi^{2}$ increases steeply for larger sizes.

(Dorschner et al. 1995). Using these values, we find that the minimum particle size is in the range $a_{\min }=\lambda / 11-\lambda / 7$. This provides very few possibilities for the particle size, which must be much smaller than $\lambda / 2 \pi$ and much larger than $\sim \lambda / 10$. We therefore consider unlikely that the transit spectrum of HD $189733 \mathrm{~b}$ can be due to particles with $k \sim 0.1$, which would require finetuning of the particle size for the Rayleigh scattering dominating between 550 and $1050 \mathrm{~nm}$.

A broader range of particle size is allowed if the imaginary part of the refraction index is significantly smaller than $\sim 0.1$. In other words, the condensate must be transparent enough for the absorption to be negligible relative to scattering. Among the possible abundant condensates, $\mathrm{MgSiO}_{3}$ presents such a property (Dorschner et al. 1995; Fortney 2005). If $\mathrm{MgSiO}_{3}$ contains no Fe atoms, the imaginary part of the refraction index is found to be $k \sim 2 \times 10^{-5}$ in the considered wavelength range. Using this value, we find $a_{\min } / \lambda=8 \times 10^{-3}$. Therefore, we conclude that $\mathrm{MgSiO}_{3}$ is the candidate species possibly responsible for the observed Rayleigh scattering in the HD 189733b spectrum, and its size must be in the range $10^{-2}-10^{-1}$ microns (see upper panel of Fig. 3).

Assuming an abundance for the $\mathrm{MgSiO}_{3}$ grains and using Eq. (3), we can evaluate the pressure at the effective planetary radius if the Rayleigh scattering by these grains dominates the transit spectrum. For $\mathrm{MgSiO}_{3}$, the refraction index is taken to be $n \sim 1.6$ in the range $550-1050 \mathrm{~nm}$ (Dorschner et al. 1995). This gives a cross section $\sigma \approx 1528 a^{6} / \lambda^{4}$. The grain abundance is obtained using solar abundance and assuming that it is limited by the available number of magnesium atoms. Thus the grains have an abundance $\xi_{\text {grain }}=2 \xi_{\mathrm{Mg}} \mu_{\mathrm{MgSiO}_{3}} /\left(\rho_{\text {grain }} 4 \pi / 3 a^{3} N m_{\mathrm{p}}\right)$, where $\xi_{\mathrm{Mg}} \approx 4 \times 10^{-5}$ is the magnesium abundance, $\mu_{\mathrm{MgSiO}_{3}}$ is about 100.4 times the mass of the proton $\left(m_{\mathrm{p}}\right), \rho_{\text {grain }}=3.2 \mathrm{~g} \mathrm{~cm}^{-3}$ is the grain density, $a$ the particle size, and $N$ Avogadro's number. Therefore we have $\xi_{\text {grain }}=1.0 \times 10^{-15}(a / 1 \mu \mathrm{m})^{-3}$. Finally, 
at $z=0$ defined by the effective radius at $\lambda \approx 750 \mathrm{~nm}$, with $T=1340 \mathrm{~K}$ we obtain a pressure $P=2 \times 10^{-3}$ bar if the size of $\mathrm{MgSiO}_{3}$ grains is about $0.01 \mu \mathrm{m}$, and $P=2 \times 10^{-6}$ bar if the size is about $0.1 \mu \mathrm{m}$. These values obtained with Eq. (3) are similar to those obtained with a general fit to the data (Fig. 3). It is noteworthy that these values are consistent with the condensation pressure of $\mathrm{MgSiO}_{3}$ which is $\sim 10^{-4}$ bar at $1400 \mathrm{~K}$ (Lodders 1999).

Finally, Rayleigh scattering by $\mathrm{MgSiO}_{3}$ is preferred to scattering by $\mathrm{H}_{2}$ to explain the observed spectrum. Indeed, molecular hydrogen requires higher pressure, at which the signature of abundant species like $\mathrm{Na}$ I, $\mathrm{KI}, \mathrm{H}_{2} \mathrm{O}$, and possibly $\mathrm{TiO}$ should overcome the Rayleigh-scattering signature, except when they have anomalously low abundances.

Because the cross section varies as $\propto a^{6}$ in the Rayleigh regime, the scattering is largely dominated by the largest particles in the size distribution. Therefore, the typical sizes quoted above must be considered as the size of the largest particles in the distribution.

\section{Discussion}

This work assumes that the whole transit spectrum from 550 to $1050 \mathrm{~nm}$ can be interpreted by the absorption by a unique species. Alternatively, there could be a combination of absorptions by various species (e.g., $\mathrm{Na}$ I, $\mathrm{KI}$, and $\mathrm{H}_{2} \mathrm{O}$ ) in different parts of the spectrum to mimic a spectrum of Rayleigh scattering with the expected atmospheric temperature. This would be coincidental, but it cannot be excluded from the present data. This question needs higher resolution data to be solved.

The temperature and pressure found above are mean values integrated along the planetary limb, where temperature is expected to vary from the pole to the equator and from one side to the other. A numerical integration of the absorption through the planetary atmosphere assuming a variation in temperature as a function of altitude of $-60 \mathrm{~K}$ per scale height (Burrows et al. 2007 ) or as a function of longitude of $350 \mathrm{~K}$ over 120 degrees (Knutson et al. 2007) shows that temperatures found by solving Eq. (1) are slightly overestimated by no more than about $3 \%$ and $2 \%$, respectively.

If the measurements of planetary radius in the $550-1050 \mathrm{~nm}$ wavelength band are extrapolated to the infrared, where Spitzer measurements are available, we can obtain a minimum effective radius due to Rayleigh scattering. From $R_{\mathrm{p}} / R_{*}=0.1564$ at $750 \mathrm{~nm}$, we extrapolate that $R_{\mathrm{p}} / R_{*}$ must be larger than 0.1539 , 0.1531 , and 0.1526 at $3.6 \mu \mathrm{m}, 5.8 \mu \mathrm{m}$, and $8.0 \mu \mathrm{m}$, respectively (with \pm 0.0003 (stat.) 0.0006 (syst.) error bars). These values are consistent with the Spitzer/IRAC measurements given by Knutson et al. (2007) and Ehrenreich et al. (2007), and with measurements by Beaulieu et al. (2007) at the limit of the upper error bars.

Rayleigh scattering has also been proposed to explain the detection of polarized scattered light (Berdyugina et al. 2007). However, this detection concerns the high upper atmosphere and cannot be directly compared to the present work on scattering deeper in the atmosphere.

Ground-based, high-resolution spectra allowed the detection of sodium in the atmosphere of HD 18973b (Redfield et al. 2008). Assuming a pressure at a given altitude, the measured absorption allows the determination of the sodium abundance. For that purpose, a fit of the sodium line shape is needed, and still has to be developed. Alternatively, by assuming the sodium abundance, measuring sodium absorption should constrain the pressure and allow for differentiating between the several carriers of the Rayleigh scattering proposed in the present paper. Presently, extinction high in the atmosphere by $\mathrm{MgSiO}_{3}$ with its low condensation pressure of $\sim 10^{-4}$ bar appears a natural explanation for the absence of broad spectral signature and is the preferred scenario.

Acknowledgements. We thank the anonymous referee for the constructive remarks. We warmly thank J.-M. Désert and G. Hébrard for fruitful discussions.

\section{References}

Ackerman, A. S., \& Marley, M. S. 2001, ApJ, 556, 872

Bates, D. R. 1984, Planet. Space Sci., 32, 785

Beaulieu, J. P., Carey, S., Ribas, I., \& Tinetti, G. 2007, ApJ [arXiv: 0711.2142] Berdyugina, S. V., Berdyugin, A. V., Fluri, D. M., \& Piirola, V. 2007, ApJL, in press [arXiv: 0712.0193]

Bouchy, F., Udry, S., Mayor, M., et al. 2005, A\&A, 444, L15

Burrows, A., Hubeny, I., Budaj, J., Knutson, H. A., \& Charbonneau, D. 2007, ApJ, 668, L171

Charbonneau, D., Brown, T. M., Noyes, R. W., \& Gilliland, R. L. 2002, ApJ, 568,377

Deming, D., Harrington, J., Seager, S., \& Richardson, L. J. 2006, ApJ, 644, 560 Dorschner, J., Begemann, B., Henning, T., Jaeger, C., \& Mutschke, H. 1995, A\&A, 300, 503

Ehrenreich, D., Tinetti, G., Lecavelier des Etangs, A., Vidal-Madjar, A., \& Selsis, F. 2006, A\&A, 448, 379

Ehrenreich, D., Hébrard, G., Lecavelier des Etangs, A., et al. 2007, ApJ, 668, L179

Fortney, J. J. 2005, MNRAS, 364, 649

Hébrard, G., \& Lecavelier des Etangs, A. 2006, A\&A, 445, 341

Hubbard, W. B., Fortney, J. J., Lunine, J. I., et al. 2001, ApJ, 560, 413

Knutson, H. A., Charbonneau, D., Allen, L. E., et al. 2007, Nature, 447, 183

Lodders, K. 1999, ApJ, 519, 793

Pont, F., Knutson, H., Gilliland, R. L., Moutou, C., \& Charbonneau, D. 2008, MNRAS, in press [arXiv:0712.1374]

Naus, H., \& Ubachs, W. 2000, Optics Lett., 25, 347

Redfield, S., Endl, M., Cochran, W. D., \& Koesterke, L. 2007, ApJL, in press [arXiv: 0712.0761]

Vidal-Madjar, A., Lecavelier des Etangs, A., Désert, J.-M., et al. 2003, Nature, 422, 143 